\title{
Analyses on Cancer Incidence and Mortality in Huai'an Area, China, 2010
}

\author{
Qin Zhang, Enchun Pan*, Yuan He, Wei Hu, Chuang Wang \\ Huai'an Center for Disease Control and Prevention, Huai'an, China \\ Email: "hypec@163.com
}

Received 24 April 2014; revised 25 May 2014; accepted 13 June 2014

Copyright (C) 2014 by authors and Scientific Research Publishing Inc.

This work is licensed under the Creative Commons Attribution International License (CC BY). http://creativecommons.org/licenses/by/4.0/

(c) (i) Open Access

\section{Abstract}

Objective: The aim of this study was to investigate the cancer incidence and mortality in Huai'an area, China, 2010. Methods: On basis of the criteria of data quality from National Central Cancer Registry (NCCR), data submitted from 8 registries in Huai'an area in 2010 were checked and evaluated. Descriptive analysis included incidence and mortality stratified by areas (urban/rural), sex, age group and cancer sites. Results: The crude incidence rate was $231.17 / 100,000$ (males 276.04/100,000, females 183.87/100,000), age-standardized incidence rate by Chinese standard population (ASIRC) was 179.53/100,000 and by World standard population (ASIRW) was 177.24/ 100,000 . The incidence rates increased rapidly in people aged 40 and over, and the peak ages of incidence were at 75 - 79 (males over 85, females at $75-79$ ). The cancer mortality rate was $151.26 / 100,000(186.06 / 100,000$ in males and 114.58/100,000 in females), age-standardized mortality rate by Chinese standard population (ASMRC) was $116.67 / 100,000$ and by World standard population (ASMRW) was $\mathbf{1 1 5 . 6 4 / 1 0 0 , 0 0 0}$. The mortality rates increased rapidly after the age of 50, reaching the peak at the ages over 85 in both males and females. Esophageal, lung, gastric, liver, colorectal, breast, pancreas, lymphoma, brain and central nervous system, cervical were the most common cancer incident sites. Esophageal, lung, liver, gastric, colorectal, pancreatic, brain and central nervous system, leukemia, breast and lymphoma were the most common cancer sites of death. Conclusions: The data quality of cancer registration is improving. As cancer burden remained high and was different between urban and rural areas, prevention and control should be implemented based on practical situation.

\section{Keywords}

Cancer, Incidence, Mortality, Huai'an Area

\footnotetext{
${ }^{*}$ Corresponding author.
} 


\section{Introduction}

Population-based cancer registry collects cancer incident cases and deaths data from covered population to describe and surveille the cancer epidemics. The cancer registration data can provide information for cancer control planning, implementation and evaluation on cancer prevention and control as also for scientific research [1]. A population-based cancer registry was established in Huai'an Center for Disease Control and Prevention in 2007, collecting information on all cancer cases from all local registries, calculating the statistical items accurately and analyzing the data of cancer incident cases and deaths from registration areas. The cancer statistics data have been broadly utilized in scientific researches, clinical trials and decision of cancer prevention and control strategies. Here, we reported on cancer incidence and mortality of 2010 in Huai'an area after uploading the cancer registry data before July 1, 2013, which was 30 months after cancer diagnosis.

\section{Materials and Methods}

\subsection{Data Source}

There 8 cancer registries in Huai'an area submitted cancer registration data in 2010, including 4 urban registries and 4 rural registries. Cancer incidence and mortality data of the 8 cancer registries were collected by Huai'an Cancer Registry in Center for Disease Control and Prevention, China. Huai'an Bureau of Statistics provided the population data. The standardized Chinese population is obtained from the fifth national population census in 2000.

\subsection{Quality Control}

Registration validity and completeness were evaluated using indices such as MV\% (proportion of morphological verification), DCO\% (percentage of cancer cases identified with death certification only) and M/I (mortality to incidence ratio). According to NCCR acceptable criterion, the following standards should be reached: MV\% higher than 66\%, DCO\% less than $15 \%$ and M/I ratio between 0.6 and 0.8 . In this paper, the overall indicators of $\mathrm{MV} \%$, DCO\%, and $\mathrm{M} / \mathrm{I}$ ratio were $67.72 \%, 0.35 \%$ and $0.65 \%$, respectively.

\subsection{Statistical Analysis}

The quality of data was assessed based on "Guideline of Chinese Cancer Registration” and referred to the criteria for "Cancer Incidence in Five Continents Volume IX" [2] by the International Agency for Cancer Registry (IACR), the International Agency for Research on Cancer (IARC). Crude incidence and mortality rates were calculated stratified by cancer type, sex, area (urban/rural) and age group, age-standardized to the 2000 Chinese population and world Segi's population. Proportion and cumulative rates were also calculated. MS-FoxPro, MSExcel, SAS and IARC issued by IARC/IACR were used for data check, evaluation and analysis [3].

\section{Results}

\subsection{Data Pooling and Quality Evaluation}

The coverage population was 5,188,287 (2,662,613 in males and 2,525,674 in females). The total cancer incident cases were 11,994 (7350 in males and 4644 in females) and deaths were 7848 (4954 in males and 2894 in females), respectively (Table 1 ).

\subsection{Incidence and Mortality of Overall Cancers}

\subsubsection{Incidence Rate}

The crude incidence rate of all cancers was 231.17/100,000 (276.04/100,000 in males and 183.87/100,000 in females). The age-standardized incidence rate by Chinese population (ASIRC) was 179.53/100,000 (226.71/ 100,000 in males and 137.08/100,000 in females) and by World standard population (ASIRW) was 177.24/ 100,000 (232.04/100,000 in males and 127.05/100,000 in females). The truncated rate (35 - 64 age years old) was 241.74/100,000. Among the patients aged 0 - 74 years, the cumulative incidence rate was $21.48 \%$. The crude cancer incidence rates and age-standardized rates in urban area were higher than those of rural area except the crude incidence in male was much higher in rural area than in urban area (Table 2). 
Table 1. Distribution for population, new case and death case in Huai'an area in 2010.

\begin{tabular}{|c|c|c|c|c|c|c|c|c|c|}
\hline \multirow{2}{*}{ Areas } & \multicolumn{3}{|c|}{ Population } & \multicolumn{3}{|c|}{ New case number } & \multicolumn{3}{|c|}{ Cancer death } \\
\hline & All & Male (\%) & Female (\%) & All & Male (\%) & Female (\%) & All & Male (\%) & Female (\%) \\
\hline All areas & $5,188,287$ & $\begin{array}{c}2,662,613 \\
(51.32)\end{array}$ & $\begin{array}{c}2,525,674 \\
(48.68)\end{array}$ & 11,994 & $\begin{array}{c}7350 \\
(61.28)\end{array}$ & $\begin{array}{c}4644 \\
(38.72)\end{array}$ & 7848 & $\begin{array}{c}4954 \\
(63.12)\end{array}$ & $\begin{array}{c}2894 \\
(36.88)\end{array}$ \\
\hline Urban & $2,636,324$ & $\begin{array}{c}1,361,315 \\
(51.64)\end{array}$ & $\begin{array}{c}1,275,009 \\
(48.36)\end{array}$ & 6192 & $\begin{array}{c}3750 \\
(60.56)\end{array}$ & $\begin{array}{c}2442 \\
(39.44)\end{array}$ & 3941 & $\begin{array}{c}2498 \\
(63.38)\end{array}$ & $\begin{array}{c}1443 \\
(36.62)\end{array}$ \\
\hline Rural & $2,551,963$ & $\begin{array}{c}1,301,298 \\
(50.99)\end{array}$ & $\begin{array}{c}1,250,665 \\
(49.01)\end{array}$ & 5802 & $\begin{array}{c}3600 \\
(62.05)\end{array}$ & $\begin{array}{c}2202 \\
(37.95)\end{array}$ & 3907 & $\begin{array}{c}2456 \\
(62.86)\end{array}$ & $\begin{array}{c}1451 \\
(37.14)\end{array}$ \\
\hline
\end{tabular}

Table 2. The incidence of cancer in Huai'an area in 2010.

\begin{tabular}{|c|c|c|c|c|c|c|c|}
\hline Areas & Genders & Cases & $\begin{array}{l}\text { Crude incidence } \\
\qquad\left(1 / 10^{5}\right)\end{array}$ & $\begin{array}{l}\text { ASIRC }^{*} \\
\left(1 / 10^{5}\right)\end{array}$ & $\begin{array}{l}\text { ASIRW }^{* *} \\
\left(1 / 10^{5}\right)\end{array}$ & $\begin{array}{l}\text { Truncated rate } \\
35-64\left(1 / 10^{5}\right)\end{array}$ & $\begin{array}{c}\text { Cumulative rate } \\
0-74(\%)\end{array}$ \\
\hline \multirow{3}{*}{ All areas } & All & 11,994 & 231.17 & 179.53 & 177.24 & 241.74 & 21.48 \\
\hline & Male & 7350 & 276.04 & 226.71 & 232.04 & 285.51 & 27.03 \\
\hline & Female & 4644 & 183.87 & 137.08 & 127.05 & 194.93 & 15.98 \\
\hline \multirow{3}{*}{ Urban } & All & 6192 & 234.87 & 187.98 & 184.87 & 249.88 & 22.43 \\
\hline & Male & 3750 & 275.47 & 235.89 & 240.21 & 292.06 & 28.07 \\
\hline & Female & 2442 & 191.53 & 145.57 & 133.91 & 203.73 & 16.90 \\
\hline \multirow{3}{*}{ Rural } & All & 5802 & 227.35 & 171.53 & 170.04 & 233.70 & 20.56 \\
\hline & Male & 3600 & 276.65 & 218.33 & 226.20 & 278.94 & 26.07 \\
\hline & Female & 2202 & 176.07 & 128.89 & 121.02 & 186.39 & 15.09 \\
\hline
\end{tabular}

*Age-standardized incidence rate (China population), ${ }^{* *}$ Age-standardized incidence rate (world population).

\subsubsection{Age-Specific Incidence Rate}

Cancer incidence was at low level before 39 years old, then increased dramatically after 40 years old and finally reached the peak after 75 years old. The pattern was similar in urban and rural areas. The peak ages of incidence in males was after 85 years old (after 75 years old in urban and after 85 years old in rural) and the peak ages of incidence in females was after 75 years old (after 75 years old in urban and after 85 years old in rural) (Figure 1).

\subsubsection{Mortality Rate}

The crude mortality in cancer registration areas was 151.26/100,000 (186.06/100, 000 in males and 114.58/ 100,000 in females). The age-standardized rate by Chinese population (ASMRC) was 116.67/100,000 (154.78/ 100,000 in males and 82.39/100,000 in females) and by World standard population (ASMRW) was 115.64/ 100,000 (157.44/100,000 in males and 73.46/100,000 in females). The truncated rate (35 - 64 age years old) was $129.95 / 100,000$. The cumulative mortality ( 0 - 74 years) rate was $13.56 \%$. Urban areas had lower crude cancer mortality of $149.49 / 100,000$ than that of rural area of 153.10/100,000, the same situation both in male and female. After age standardization, the mortality rate in urban area was higher than that in rural area and both in males and females (Table 3).

\subsubsection{Age-Specific Mortality}

Cancer mortality was at low level before 49 years old, then increased dramatically after 50 years old and finally reached the peak after 85 years old. The pattern was similar in urban and rural areas. The peak ages of mortality in both male and female were after 85 years old (Figure 2).

\subsection{Incidence and Mortality for Major Cancers}

\subsubsection{Cancer Incidence Rates for the 10 Most Common Cancers}

Esophagus cancer was the most common cancer in Huai'an area, China, followed by lung cancer, stomach cancer, liver cancer and colorectal cancer. The most frequently diagnosed cancers in males were esophageal cancer, 


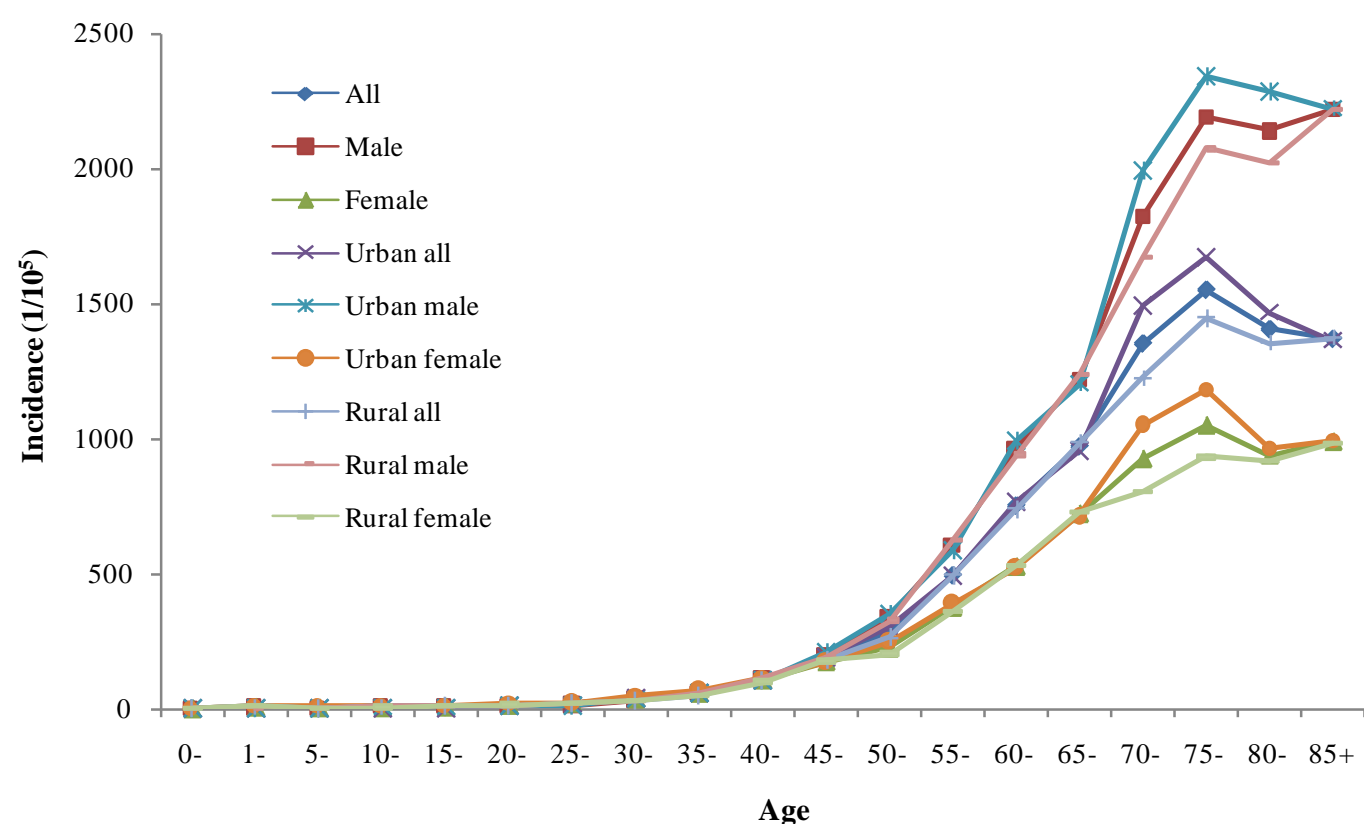

Figure 1. Age-specific incidence rate for all cancers in Huai' an area in $2010\left(1 / 10^{5}\right)$.

Table 3. The mortality of cancer in Huai'an area in 2010.

\begin{tabular}{cccccccc}
\hline Areas & Genders & Cases & $\begin{array}{c}\text { Crude incidence } \\
\left(1 / 10^{5}\right)\end{array}$ & $\begin{array}{c}\text { ASMRC }^{*} \\
\left(1 / 10^{5}\right)\end{array}$ & $\begin{array}{c}\text { ASMRW }^{* *} \\
\left(1 / 10^{5}\right)\end{array}$ & $\begin{array}{c}\text { Truncated rate } \\
35-64\left(1 / 10^{5}\right)\end{array}$ & $\begin{array}{c}\text { Cumulative rate } \\
0-74(\%)\end{array}$ \\
\hline \multirow{4}{*}{ All areas } & All & 7848 & 151.26 & 116.67 & 115.64 & 129.95 & 13.56 \\
& Male & 4954 & 186.06 & 154.78 & 157.44 & 167.59 & 17.80 \\
& Female & 2894 & 114.58 & 82.39 & 73.46 & 89.92 & 9.36 \\
& All & 3941 & 149.49 & 119.27 & 118.40 & 134.07 & 14.14 \\
\multirow{4}{*}{ Urban } & Male & 2498 & 183.50 & 158.92 & 160.46 & 171.96 & 18.54 \\
& Female & 1443 & 113.18 & 84.01 & 74.88 & 93.09 & 9.81 \\
& All & 3907 & 153.10 & 114.31 & 113.30 & 126.01 & 13.00 \\
& Male & 2456 & 188.73 & 151.11 & 156.75 & 163.36 & 17.10 \\
& Female & 1451 & 116.02 & 80.95 & 72.76 & 87.02 & 8.93 \\
\hline
\end{tabular}

"Age-standardized mortality rate (China population), ${ }^{* *}$ Age-standardized mortality rate (world population).

lung cancer, stomach cancer, liver cancer and colorectal cancer. And the most frequently diagnosed cancers in females were esophageal cancer, stomach cancer, lung cancer, breast cancer and liver cancer. The 10 most common cancers accounted for $88.10 \%$ of all new cases with $91.17 \%$ in males and $86.63 \%$ in females, respectively (Table 4).

\subsubsection{Cancer Incidence of the 10 Most Common Cancers in Urban Areas}

In urban area, esophageal cancer was the most frequently diagnosed cancers, followed by stomach cancer, lung cancer, liver cancer, and colorectal cancer. The most frequently diagnosed cancers in males were esophageal cancer, stomach cancer, lung cancer, liver cancer and colorectal cancer. And the most frequently diagnosed cancers in females were esophageal cancer, stomach cancer, breast cancer, lung cancer and liver cancer. The 10 most common cancer accounted for $87.45 \%$ (91.68\% in males and $86.28 \%$ in females) of all cancers (Table 5).

\subsubsection{Cancer Incidence of the 10 Most Common Cancers in Rural Areas}

In rural area, the most frequently diagnosed cancers were esophageal cancer, lung cancer, stomach cancer, liver cancer and colorectal cancer. The most common sites of cancer were esophagus, lung, stomach, liver and colon- 


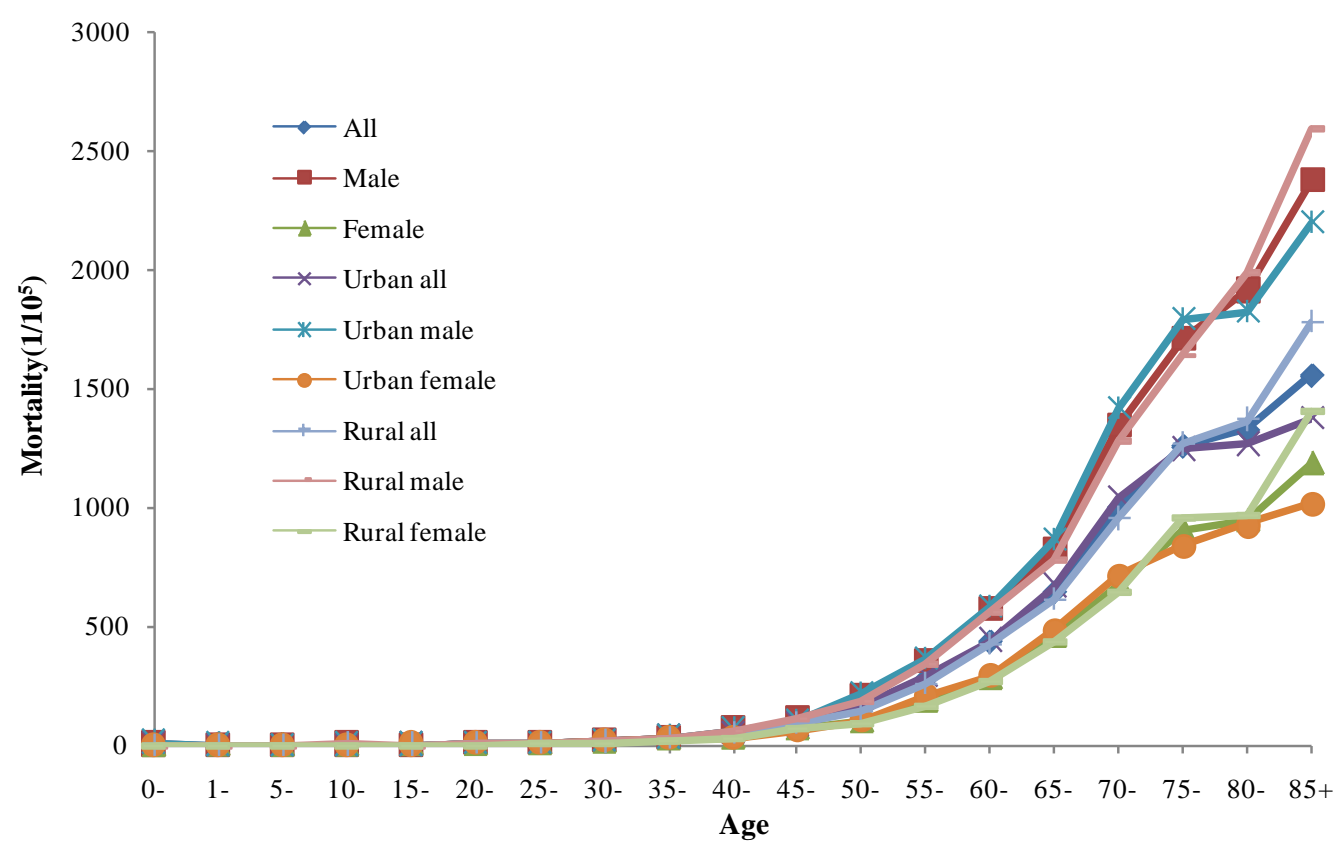

Figure 2. Age-specific mortality rate for all cancers in Huai'an area in $2010\left(1 / 10^{5}\right)$.

Table 4. The 10 most common cancer incidence rates in Huai'an area in 2010.

\begin{tabular}{|c|c|c|c|c|c|c|c|c|c|c|c|c|}
\hline \multirow[b]{2}{*}{ Rank } & \multicolumn{4}{|c|}{ All } & \multicolumn{4}{|c|}{ Male } & \multicolumn{4}{|c|}{ Female } \\
\hline & Site & $\begin{array}{c}\text { Crude } \\
\text { incidence } \\
\left(1 / 10^{5}\right)\end{array}$ & $\begin{array}{c}\text { Ratio* } \\
(\%)\end{array}$ & $\begin{array}{c}\text { ASIRC** } \\
\left(1 / 10^{5}\right)\end{array}$ & Site & $\begin{array}{c}\text { Crude } \\
\text { incidence } \\
\left(1 / 10^{5}\right)\end{array}$ & $\begin{array}{c}\text { Ratio }^{*} \\
(\%)\end{array}$ & $\begin{array}{c}\text { ASIRC }^{* *} \\
\left(1 / 10^{5}\right)\end{array}$ & Site & $\begin{array}{c}\text { Crude } \\
\text { incidence } \\
\left(1 / 10^{5}\right)\end{array}$ & $\begin{array}{c}\text { Ratio }^{*} \\
(\%)\end{array}$ & $\begin{array}{l}\text { ASIRC ** }_{\left(1 / 10^{5}\right)}\end{array}$ \\
\hline 1 & Esophagus & 67.88 & 29.36 & 51.57 & Esophagus & 83.68 & 30.31 & 67.80 & Esophagus & 51.23 & 27.86 & 36.49 \\
\hline 2 & Lung & 35.68 & 15.43 & 27.21 & Lung & 48.41 & 17.54 & 39.66 & Stomach & 22.33 & 12.14 & 16.15 \\
\hline 3 & Stomach & 34.73 & 15.02 & 26.57 & Stomach & 46.50 & 16.84 & 37.86 & Lung & 22.25 & 12.10 & 15.95 \\
\hline 4 & Liver & 27.22 & 11.77 & 21.22 & Liver & 39.70 & 14.38 & 32.13 & Breast & 18.41 & 10.01 & 14.80 \\
\hline 5 & Colon-rectu & 12.35 & 5.34 & 9.64 & Colon-rectu & 14.16 & 5.13 & 11.66 & Liver & 14.06 & 7.64 & 10.32 \\
\hline 6 & Breast & 9.10 & 3.94 & 7.39 & Pancreas & 5.52 & 2.00 & 4.61 & Colon-rectu & 10.45 & 5.68 & 7.84 \\
\hline 7 & Pancreas & 4.57 & 1.98 & 3.51 & Leukemia & 4.88 & 1.77 & 4.76 & Cervix uteri & 7.80 & 4.22 & 6.68 \\
\hline 8 & Leukemia & 4.32 & 1.87 & 4.06 & Brain (CNS) & 4.09 & 1.48 & 3.68 & Uterus & 5.07 & 2.76 & 3.89 \\
\hline 9 & Brain(CNS) & 4.05 & 1.75 & 3.33 & Bladder & 3.49 & 1.27 & 2.82 & Brain(CNS) & 4.00 & 2.17 & 3.03 \\
\hline \multirow[t]{2}{*}{10} & Cervix uteri & 3.80 & 1.63 & 3.30 & Nasopharynx & 3.46 & 1.25 & 2.89 & Leukemia & 3.72 & 2.02 & 3.40 \\
\hline & Top 10 & 203.70 & 88.10 & 157.79 & Top 10 & 253.88 & 91.97 & 207.88 & Top 10 & 159.32 & 86.63 & 118.55 \\
\hline
\end{tabular}

${ }^{*}$ Constituent ratio, ${ }^{* *}$ Age-standardized incidence rate (China population).

rectum in males, while were esophagus, lung, stomach, breast and liver cancer in females. The 10 most common cancers accounted for $88.80 \%$ (92.74\% in males and $87.24 \%$ in females) of all registered new cases (Table 6).

\subsubsection{Cancer Mortality Rates for the 10 Most Common Cancers}

Esophagus cancer was the most common cancer of death in Huai'an area, China, followed by lung cancer, liver cancer, stomach cancer and colorectal cancer. In males, esophageal cancer was the leading cause followed by lung cancer, liver cancer, stomach cancer and colorectal cancer, while in females, esophageal cancer was still the leading cause followed by lung cancer, stomach cancer, liver cancer and breast cancer. The 10 most common cancers accounted for $91.32 \%$ of all cases with $94.17 \%$ in males and $88.87 \%$ in females, respectively (Table 7). 
Table 5. The 10 most common cancer incidence rates in urban areas in 2010.

\begin{tabular}{|c|c|c|c|c|c|c|c|c|c|c|c|c|}
\hline \multirow[b]{2}{*}{ Rank } & \multicolumn{4}{|c|}{ All } & \multicolumn{4}{|c|}{ Male } & \multicolumn{4}{|c|}{ Female } \\
\hline & Site & $\begin{array}{c}\text { Crude } \\
\text { incidence } \\
\left(1 / 10^{5}\right)\end{array}$ & $\begin{array}{c}\text { Ratio }^{*} \\
(\%)\end{array}$ & $\begin{array}{c}\operatorname{ASIRC}^{* *} \\
\left(1 / 10^{5}\right)\end{array}$ & Site & $\begin{array}{c}\text { Crude } \\
\text { incidence } \\
\left(1 / 10^{5}\right)\end{array}$ & $\begin{array}{c}\text { Ratio* } \\
(\%)\end{array}$ & $\begin{array}{c}\operatorname{ASIRC}^{* *} \\
\left(1 / 10^{5}\right)\end{array}$ & Site & $\begin{array}{l}\text { Crude } \\
\text { incidence } \\
\left(1 / 10^{5}\right)\end{array}$ & $\begin{array}{c}\text { Ratio* } \\
(\%)\end{array}$ & $\begin{array}{c}\text { ASIRC }^{* *} \\
\left(1 / 10^{5}\right)\end{array}$ \\
\hline 1 & Esophagus & 69.76 & 29.70 & 54.81 & Esophagus & 82.71 & 30.03 & 69.91 & Esophagus & 55.92 & 29.20 & 40.62 \\
\hline 2 & Stomach & 34.29 & 14.60 & 27.24 & Stomach & 45.91 & 16.67 & 39.48 & Stomach & 21.88 & 11.43 & 16.14 \\
\hline 3 & Lung & 33.38 & 14.21 & 26.33 & Lung & 44.81 & 16.27 & 38.42 & Breast & 21.88 & 11.43 & 17.51 \\
\hline 4 & Liver & 28.30 & 12.05 & 22.49 & Liver & 41.87 & 15.20 & 34.55 & Lung & 21.18 & 11.06 & 15.58 \\
\hline 5 & Colon-rectu & 12.33 & 5.25 & 9.81 & Colon-rectu & 14.18 & 5.15 & 12.01 & Liver & 13.80 & 7.21 & 10.56 \\
\hline 6 & Breast & 10.66 & 4.54 & 8.66 & Leukemia & 5.80 & 2.19 & 5.83 & Colon-rectu & 10.35 & 5.41 & 7.85 \\
\hline 7 & Leukemia & 5.01 & 2.18 & 4.76 & Pancreas & 5.95 & 2.03 & 4.93 & Cervix uteri & 6.59 & 3.44 & 5.16 \\
\hline 8 & Pancreas & 4.67 & 1.99 & 3.74 & Bladder & 4.11 & 1.49 & 3.66 & Uterus & 5.80 & 3.03 & 4.47 \\
\hline 9 & Brain (CNS) & 3.72 & 1.58 & 3.19 & Brain (CNS) & 3.97 & 1.44 & 3.71 & Leukemia & 4.16 & 2.17 & 3.63 \\
\hline 10 & Cervix uteri & 3.19 & 1.36 & 2.56 & Nasopharynx & 3.38 & 1.23 & 2.96 & Pancreas & 3.69 & 1.92 & 2.74 \\
\hline & Top 10 & 205.29 & 87.45 & 163.59 & Top 10 & 252.69 & 91.68 & 215.47 & Top 10 & 165.25 & 86.28 & 124.26 \\
\hline
\end{tabular}

*Constituent ratio, ${ }^{* *}$ Age-standardized incidence rate (China population).

Table 6. The 10 most common cancer incidence rates in rural areas in 2010.

\begin{tabular}{|c|c|c|c|c|c|c|c|c|c|c|c|c|}
\hline \multirow[b]{2}{*}{ Rank } & \multicolumn{4}{|c|}{ All } & \multicolumn{4}{|c|}{ Male } & \multicolumn{4}{|c|}{ Female } \\
\hline & Site & $\begin{array}{c}\text { Crude } \\
\text { incidence } \\
\left(1 / 10^{5}\right)\end{array}$ & $\begin{array}{c}\text { Ratio* } \\
(\%)\end{array}$ & $\begin{array}{c}\text { ASIRC }^{* *} \\
\left(1 / 10^{5}\right)\end{array}$ & Site & $\begin{array}{c}\text { Crude } \\
\text { Incidence } \\
\left(1 / 10^{5}\right)\end{array}$ & $\begin{array}{c}\text { Ratio* } \\
\text { (\%) }\end{array}$ & $\begin{array}{c}\text { ASIRC }^{* *} \\
\left(1 / 10^{5}\right)\end{array}$ & Site & $\begin{array}{c}\text { Crude } \\
\text { incidence } \\
\left(1 / 10^{5}\right)\end{array}$ & $\begin{array}{c}\text { Ratio* } \\
\text { (\%) }\end{array}$ & $\begin{array}{c}\text { ASIRC }^{* *} \\
\left(1 / 10^{5}\right)\end{array}$ \\
\hline 1 & Esophagus & 65.95 & 29.01 & 48.50 & Esophagus & 84.68 & 30.61 & 65.66 & Esophagus & 46.46 & 26.39 & 32.63 \\
\hline 2 & Lung & 38.05 & 16.74 & 28.15 & Lung & 52.18 & 18.86 & 41.17 & Lung & 23.35 & 13.26 & 16.31 \\
\hline 3 & Stomach & 35.19 & 15.48 & 26.07 & Stomach & 47.11 & 17.03 & 36.51 & Stomach & 22.79 & 12.94 & 16.31 \\
\hline 4 & Liver & 26.10 & 11.48 & 20.01 & Liver & 37.42 & 13.53 & 29.89 & Breast & 14.87 & 8.45 & 12.04 \\
\hline 5 & Colon-rectu & 12.38 & 5.45 & 9.44 & Colon-rectu & 14.14 & 5.11 & 11.31 & Liver & 14.31 & 8.13 & 10.10 \\
\hline 6 & Breast & 7.48 & 3.29 & 6.08 & Pancreas & 5.46 & 1.97 & 4.33 & Colon-rectu & 10.55 & 5.99 & 7.76 \\
\hline 7 & Pancreas & 4.47 & 1.96 & 3.31 & Brain (CNS) & 4.23 & 1.53 & 3.65 & Cervix uteri & 8.96 & 5.09 & 7.08 \\
\hline 8 & Cervix uteri & 4.39 & 1.93 & 3.54 & Leukemia & 3.69 & 1.33 & 3.42 & Brain (CNS) & 4.56 & 2.59 & 3.31 \\
\hline 9 & Brain (CNS) & 4.39 & 1.93 & 3.46 & Nasopharynx & 3.53 & 1.28 & 2.78 & Uterus & 4.32 & 2.45 & 3.32 \\
\hline \multirow[t]{2}{*}{10} & Leukemia & 3.49 & 1.53 & 3.09 & Lymphoma & 3.38 & 1.22 & 2.71 & Pancreas & 3.44 & 1.95 & 2.28 \\
\hline & Tор 10 & 201.88 & 88.80 & 151.64 & Top 10 & 255.82 & 92.47 & 201.43 & Top 10 & 153.60 & 87.24 & 111.14 \\
\hline
\end{tabular}

${ }^{*}$ Constituent ratio, ${ }^{* *}$ Age-standardized incidence rate (China population).

\subsubsection{Cancer Mortality of the 10 Most Common Cancers in Urban Areas}

In urban area, esophageal cancer was the leading cause of cancer death, followed by lung cancer, stomach cancer, liver cancer and colorectal cancer. And the most frequently cancers in males were esophageal cancer, lung cancer, liver cancer, stomach cancer and colorectal cancer while in females were esophageal cancer, stomach cancer, lung cancer, liver cancer and breast cancer. The top 10 cancers accounted for 92.13\% (95.08\% for males and $89.60 \%$ for females) of all mortality induced by malignant tumors (Table 8 ).

\subsubsection{Cancer Mortality of the 10 Most Common Cancers in Rural Areas}

In rural area, esophageal cancer was the leading cause of cancer death, followed by lung cancer, liver cancer, stomach cancer and colorectal cancer. And the most frequently cancers in males were esophageal cancer, lung cancer, liver cancer, stomach cancer and colorectal cancer while in females were esophageal cancer, lung cancer, stomach cancer, liver cancer and breast cancer. The top 10 cancers accounted for $90.79 \%$ (93.61\% for males and 88.35\% for females) of all mortality induced by malignant tumors (Table 9). 
Table 7. The 10 most common cancer mortality rates in Huai'an area in 2010.

\begin{tabular}{|c|c|c|c|c|c|c|c|c|c|c|c|c|}
\hline \multirow[b]{2}{*}{ Rank } & \multicolumn{4}{|c|}{ All } & \multicolumn{4}{|c|}{ Male } & \multicolumn{4}{|c|}{ Female } \\
\hline & Site & $\begin{array}{c}\text { Crude } \\
\text { mortality } \\
\left(1 / 10^{5}\right)\end{array}$ & $\begin{array}{c}\text { Ratio }^{*} \\
(\%)\end{array}$ & $\begin{array}{c}\operatorname{ASMRC}^{* *} \\
\left(1 / 10^{5}\right)\end{array}$ & Site & $\begin{array}{c}\text { Crude } \\
\text { mortality } \\
\left(1 / 10^{5}\right)\end{array}$ & $\begin{array}{c}\text { Ratio }^{*} \\
(\%)\end{array}$ & $\begin{array}{c}\operatorname{ASMRC}^{* *} \\
\left(1 / 10^{5}\right)\end{array}$ & Site & $\begin{array}{c}\text { Crude } \\
\text { mortality } \\
\left(1 / 10^{5}\right)\end{array}$ & $\begin{array}{c}\text { Ratio }^{*} \\
(\%)\end{array}$ & $\begin{array}{c}\text { ASMRC }^{* *} \\
\left(1 / 10^{5}\right)\end{array}$ \\
\hline 1 & Esophagus & 48.63 & 32.15 & 36.97 & Esophagus & 57.99 & 31.17 & 48.17 & Esophagus & 38.76 & 33.83 & 27.01 \\
\hline 2 & Lung & 25.42 & 16.81 & 19.33 & Lung & 34.21 & 18.39 & 28.35 & Lung & 16.15 & 14.10 & 11.34 \\
\hline 3 & Liver & 22.47 & 14.86 & 17.49 & Liver & 32.45 & 17.44 & 26.42 & Stomach & 15.16 & 13.23 & 10.70 \\
\hline 4 & Stomach & 22.38 & 14.79 & 17.16 & Stomach & 29.22 & 15.70 & 24.32 & Liver & 11.96 & 10.44 & 8.72 \\
\hline 5 & Colon-rectu & 5.61 & 3.71 & 4.35 & Colon-rectu & 6.99 & 3.75 & 5.86 & Breast & 5.23 & 4.56 & 4.04 \\
\hline 6 & Pancreas & 3.57 & 2.36 & 2.74 & Pancreas & 4.36 & 2.34 & 3.69 & Colon-rectu & 4.16 & 3.63 & 3.06 \\
\hline 7 & Brain (CNS) & 3.03 & 2.00 & 2.40 & Leukemia & 3.57 & 1.92 & 3.33 & Pancreas & 2.73 & 2.38 & 1.94 \\
\hline 8 & Leukemia & 2.99 & 1.98 & 2.64 & Brain (CNS) & 3.38 & 1.82 & 2.86 & Brain (CNS) & 2.65 & 2.32 & 1.98 \\
\hline 9 & Breast & 2.54 & 1.68 & 1.99 & Lymphoma & 1.58 & 0.85 & 1.31 & Cervix uteri & 2.65 & 2.32 & 1.94 \\
\hline \multirow[t]{2}{*}{10} & Lymphoma & 1.50 & 0.99 & 1.17 & Bone & 1.46 & 0.79 & 1.21 & Leukemia & 2.38 & 2.07 & 1.92 \\
\hline & Top 10 & 138.14 & 91.32 & 106.25 & Top 10 & 175.20 & 94.17 & 145.53 & Top 10 & 101.83 & 88.87 & 72.65 \\
\hline
\end{tabular}

*Constituent ratio, ${ }^{* *}$ Age-standardized mortality rate (China population).

Table 8. The 10 most common cancer mortality rates in urban areas in 2010.

\begin{tabular}{|c|c|c|c|c|c|c|c|c|c|c|c|c|}
\hline \multirow[b]{2}{*}{ Rank } & \multicolumn{4}{|c|}{ All } & \multicolumn{4}{|c|}{ Male } & \multicolumn{4}{|c|}{ Female } \\
\hline & Site & $\begin{array}{l}\text { Crude } \\
\text { mortality } \\
\left(1 / 10^{5}\right)\end{array}$ & $\begin{array}{c}\text { Ratio* } \\
(\%)\end{array}$ & $\begin{array}{l}\text { ASMRC }^{* *} \\
\left(1 / 10^{5}\right)\end{array}$ & Site & $\begin{array}{c}\text { Crude } \\
\text { mortality } \\
\left(1 / 10^{5}\right)\end{array}$ & $\begin{array}{c}\text { Ratio* } \\
(\%)\end{array}$ & $\begin{array}{c}\text { ASMRC }^{* *} \\
\left(1 / 10^{5}\right)\end{array}$ & Site & $\begin{array}{l}\text { Crude } \\
\text { mortality } \\
\left(1 / 10^{5}\right)\end{array}$ & $\begin{array}{c}\text { Ratio* } \\
(\%)\end{array}$ & $\begin{array}{c}\operatorname{ASMRC}^{* *} \\
\left(1 / 10^{5}\right)\end{array}$ \\
\hline 1 & Esophagus & 52.72 & 35.27 & 41.47 & Esophagus & 62.44 & 34.03 & 53.70 & Esophagus & 42.35 & 37.42 & 30.34 \\
\hline 2 & Lung & 23.18 & 15.50 & 18.29 & Lung & 31.29 & 17.05 & 27.24 & Stomach & 14.98 & 13.24 & 10.94 \\
\hline 3 & Stomach & 22.72 & 15.20 & 18.17 & Liver & 30.12 & 16.41 & 25.19 & Lung & 14.51 & 12.82 & 10.53 \\
\hline 4 & Liver & 20.26 & 13.55 & 16.11 & Stomach & 29.97 & 16.33 & 26.34 & Liver & 9.73 & 8.59 & 7.37 \\
\hline 5 & Colon-rectu & 5.58 & 3.73 & 4.48 & Colon-rectu & 6.76 & 3.68 & 5.81 & Breast & 6.20 & 5.47 & 4.87 \\
\hline 6 & Pancreas & 3.34 & 2.23 & 2.64 & Pancreas & 4.19 & 2.28 & 3.66 & Colon-rectu & 4.31 & 3.81 & 3.35 \\
\hline 7 & Breast & 3.00 & 2.00 & 2.39 & Leukemia & 3.60 & 1.96 & 3.50 & Pancreas & 2.43 & 2.15 & 1.81 \\
\hline 8 & Leukemia & 2.88 & 1.93 & 2.70 & Brain (CNS) & 3.16 & 1.72 & 2.81 & Brain (CNS) & 2.43 & 2.15 & 1.97 \\
\hline 9 & Brain (CNS) & 2.81 & 1.88 & 2.37 & Bladder & 1.62 & 0.88 & 1.48 & Uterus & 2.35 & 2.08 & 1.85 \\
\hline \multirow[t]{2}{*}{10} & Bladder & 1.25 & 0.84 & 1.00 & Bone & 1.32 & 0.72 & 1.15 & Leukemia & 2.12 & 1.87 & 1.85 \\
\hline & Top 10 & 137.73 & 92.13 & 109.63 & Top 10 & 174.46 & 95.08 & 150.90 & Top 10 & 101.41 & 89.60 & 74.89 \\
\hline
\end{tabular}

${ }^{*}$ Constituent ratio, ${ }^{* *}$ Age-standardized mortality rate (China population).

\section{Discussion}

Huai'an city is a prefecture-level city in the middle Jiangsu province which was with high cancer incidence in China. Since a population-based cancer registry was established in Huai'an Center for Disease Control and Prevention in 2007, there were 8 cancer registries which covered the whole city with 6 of them supported by central finance. Huai'an cancer registry has focused on improving data quantity as well as quality and has provided important information on cancer patterns over the seven years, which may be used in cancer prevention and control programs. The MV\% for all cancer sites was $67.72 \%$ which was more than $66 \%$. The DCO\% was $0.35 \%$ which was less than $15 \%$. And the M/I ratio was 0.65 which was between 0.6 and 0.8 . The data was identified qualified. The pooled data was valid and could represent cancer burden in whole city level. The statistics showed that cancer incidence and mortality in 2010 were very close to the figures in the near three years which indicated that the rates are reasonably stable [4]. According to the whole country level, the age-standardized cancer incidence rate (ASIRC, 2000) was nearly the same level, but the age-standardized cancer mortality rate (ASMRC, 2000) 
Table 9. The 10 most common cancer mortality rates in rural areas in 2010.

\begin{tabular}{|c|c|c|c|c|c|c|c|c|c|c|c|c|}
\hline \multirow[b]{2}{*}{ Rank } & \multicolumn{4}{|c|}{ All } & \multicolumn{4}{|c|}{ Male } & \multicolumn{4}{|c|}{ Female } \\
\hline & Site & $\begin{array}{c}\text { Crude } \\
\text { mortality } \\
\left(1 / 10^{5}\right)\end{array}$ & $\begin{array}{c}\text { Ratio* } \\
\text { (\%) }\end{array}$ & $\begin{array}{c}\text { ASMRC }^{* *} \\
\left(1 / 10^{5}\right)\end{array}$ & Site & $\begin{array}{c}\text { Crude } \\
\text { mortality } \\
\left(1 / 10^{5}\right)\end{array}$ & $\begin{array}{c}\text { Ratio* } \\
(\%)\end{array}$ & $\begin{array}{c}\text { ASMRC }^{* *} \\
\left(1 / 10^{5}\right)\end{array}$ & Site & $\begin{array}{c}\text { Crude } \\
\text { mortality } \\
\left(1 / 10^{5}\right)\end{array}$ & $\begin{array}{c}\text { Ratio* } \\
(\%)\end{array}$ & $\begin{array}{c}\text { ASMRC }^{* *} \\
\left(1 / 10^{5}\right)\end{array}$ \\
\hline 1 & Esophagus & 44.40 & 29.00 & 32.65 & Esophagus & 53.33 & 28.26 & 42.72 & Esophagus & 35.10 & 30.25 & 23.88 \\
\hline 2 & Lung & 27.74 & 18.12 & 20.38 & Lung & 37.27 & 19.75 & 29.60 & Lung & 17.83 & 15.37 & 12.10 \\
\hline 3 & Liver & 24.77 & 16.18 & 18.87 & Liver & 34.89 & 18.49 & 27.76 & Stomach & 15.35 & 13.23 & 10.56 \\
\hline 4 & Stomach & 22.02 & 14.38 & 16.25 & Stomach & 28.43 & 15.07 & 22.47 & Liver & 14.23 & 12.27 & 10.03 \\
\hline 5 & Colon-rectu & 5.64 & 3.69 & 4.19 & Colon-rectu & 7.22 & 3.83 & 5.88 & Breast & 4.24 & 3.65 & 4.24 \\
\hline 6 & Pancreas & 3.80 & 2.48 & 2.84 & Pancreas & 4.53 & 2.40 & 3.74 & Colon-rectu & 4.00 & 3.45 & 2.74 \\
\hline 7 & Brain (CNS) & 3.25 & 2.12 & 2.42 & Brain (CNS) & 3.61 & 1.91 & 2.90 & Cervix uteri & 3.20 & 2.76 & 2.28 \\
\hline 8 & Leukemia & 3.10 & 2.02 & 2.63 & Leukemia & 3.53 & 1.87 & 3.19 & Pancreas & 3.04 & 2.62 & 2.07 \\
\hline 9 & Lymphoma & 2.19 & 1.43 & 1.66 & Lymphoma & 2.07 & 1.10 & 1.67 & Brain (CNS) & 2.88 & 2.48 & 1.98 \\
\hline \multirow[t]{2}{*}{10} & Breast & 2.08 & 1.36 & 1.60 & Nasopharynx & 1.77 & 0.94 & 1.35 & Leukemia & 2.64 & 2.27 & 2.04 \\
\hline & Tор 10 & 138.99 & 90.79 & 103.48 & Top 10 & 176.67 & 93.61 & 141.28 & Top 10 & 102.51 & 88.35 & 71.93 \\
\hline
\end{tabular}

*Constituent ratio, ${ }^{* *}$ Age-standardized mortality rate (China population).

was a little higher than national level [5]. In urban area, both the age-standardized incidence rate and mortality rate were higher than national level [5]. In this study, all the standardized rates were higher in urban area than in rural area. Not only cancer incidence rate but also mortality rate was higher in male than in female for both in urban and rural. Cancer incidence and mortality both reached the peak rates at old age group. The pattern was similar between urban and rural areas. That means the old people were the key population for cancer prevention and control.

The top most common cancer sites in Huai'an area were esophagus, lung, stomach, liver, colon-rectum (breast in females). The incidence and mortality rates of esophagus were much higher than national and international level [5]-[7]. Both in urban area and in rural area, esophageal cancer was the leading cancer in cancer incidence and mortality for both men and women, while the esophageal cancer rank was the fifth in cancer incidence and fourth in mortality compared with the report of cancer incidence from China cancer registration areas in 2009 and was the eighth in cancer incidence and sixth in mortality compared with the report from WHO [5]-[7]. Esophageal cancer is the result of both effect of environmental factors and genetic factors. And lifestyle is one of the most important influencing factors, such as tobacco smoking, alcohol consumption, ingestion of hot foods and drinks, fast eating speed, nutrition deficiency [8]-[12]. To our investigation, people are used to more preserved foods, such as pickled vegetables, salted fish, bacon and so on. And excessive alcohol consumption may be another problem. Therefore, health education/promotion, behavioral intervention, early diagnosis/treatment in population are urgently anticipated in Huai'an area, and appeal keeping away from the risk factors of esophageal cancer. Following esophagus caner, lung cancer was the second one in cancer incidence and mortality. In females, breast cancer is a large disease burden especially in urban which was the third diagnosed cancer. Although in men prostate cancer is the second most common cancer and the fifth leading cause of death in the world, it has very low incidence and mortality rates in our city.

\section{Conclusion}

In conclusion, cancer is severely threatening people's health, and esophagus, lung, stomach, liver and colon-rectum cancers (breast cancer) are the major burden of cancer. Cancer prevention and control program should focus on these cancers, especially esophageal cancer. The comprehensive intervention methods including health education/promotion, behavioral intervention, such as tobacco control and healthy lifestyle, should be enhanced and especially early diagnosis/treatment for esophageal cancer should be executed in the long run.

\section{Acknowledgements}

We gratefully acknowledged all 8 cancer registries in Huai'an area providing cancer statistics, data collection, 
sorting, verification and database creation. The authors assume full responsibility for analyses and interpretation of these data.

\section{Disclosure}

The authors declare no conflicts of interest.

\section{References}

[1] Parkin, D.M. (2006) The Evolution of the Population-Based Cancer Registry. Natural Reviews of Cancer, 6, 603-612. http://dx.doi.org/10.1038/nrc1948

[2] Curado, M.P., Edwards, B., Shin, H.R., et al. (2008) Cancer Incidence in Five Continents. Vol. IX. IARC Scientific Publications No. 160, IARC, Lyon.

[3] Ferlay, J. (2006) The IARC crg Tools Programs. IACR, Lyon. http://www.iacr.com.fr/iarccrgtools.htm

[4] Yuan, G.J., Li, Q.W., Du, Y.X., et al. (2012) Analyses on Cancer Incidence and Mortality in Huai’an Area, China, from 2009 to 2011. Chinese-German Journal of Clinical Oncology, 11, 504-507. http://dx.doi.org/10.1007/s10330-012-1035-4

[5] Chen, W., Zheng, R., Zhang, S., et al. (2014) Annual Report on Status of Cancer in China, 2010. Chinese Journal of Cancer Research, 26, 48-58.

[6] Chen, W., Zheng, R., Zhang, S., et al. (2013) Report of Incidence and Mortality in China Cancer Registries, 2009. Chinese Journal of Cancer Research, 25, 10-21.

[7] World Health Organization (2014) GLOBOCAN2012: Estimated Cancer Incidence, Mortality and Prevalence Worldwide in 2012. http://globocan.iarc.fr/Default.aspx

[8] Wu, M., Zhang, Z.F., Kampman, E., et al. (2011) Does Family History of Cancer Modify the Effects of Lifestyle Risk Factors on Esophageal Cancer? A Population-Based Case-Control Study in China. International Journal of Cancer, 128, 2147-2157. http://dx.doi.org/10.1002/ijc.25532

[9] Wu, M., Van’t Veer, P., Zhang, Z.F., et al. (2011) A Large Proportion of Esophageal Cancer Cases and the Incidence Difference between Regions Are Attributable to Lifestyle Risk Factors in China. Cancer Letters, 308, 189-196. http://dx.doi.org/10.1016/j.canlet.2011.05.003

[10] Wang, J.B., Fan, J.H., Liang, H., et al. (2012) Attributable Causes of Esophageal Cancer Incidence and Mortality in China. PLoS One, 7, e42281. http://dx.doi.org/10.1371/journal.pone.0042281

[11] Liu, M., Su, M., Tian, D.P., et al. (2010) Heredity, Diet and Lifestyle as Determining Risk Factors for the Esophageal Cancer on Nanao Island in Southern China. Family Cancer, 9, 229-238. http://dx.doi.org/10.1007/s10689-009-9300-6

[12] Wu, M., Zhao, J.K., Hu, X.S., et al. (2006) Association of Smoking, Alcohol Drinking and Dietary Factors with Esophageal Cancer in High- and Low-Risk Areas of Jiangsu Province, China. World Journal of Gastroenterology, 12, 1686-1693. 
Scientific Research Publishing (SCIRP) is one of the largest Open Access journal publishers. It is currently publishing more than 200 open access, online, peer-reviewed journals covering a wide range of academic disciplines. SCIRP serves the worldwide academic communities and contributes to the progress and application of science with its publication.

Other selected journals from SCIRP are listed as below. Submit your manuscript to us via either submit@scirp.org or Online Submission Portal.
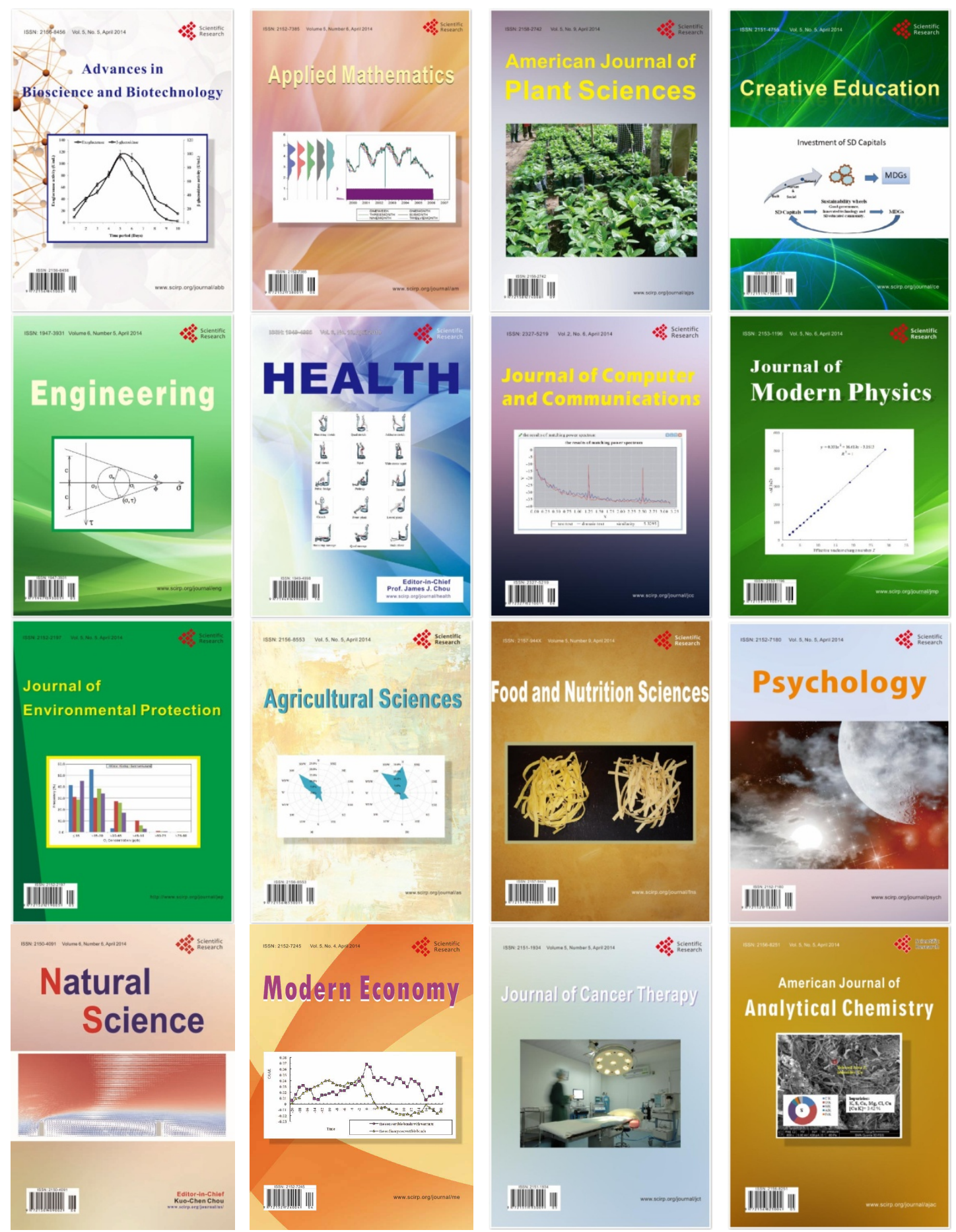